\title{
Functional and Molecular Characterization of C91S Mutation in the Second Epidermal Growth Factor-like Domain of Factor VII
}

\author{
Amir Mashayekhi ${ }^{1}$, Shirin Shahbazi ${ }^{1 *}$, Mirdavood Omrani ${ }^{2}$ \\ ${ }^{1}$ Department of Medical Genetics, Faculty of Medical Sciences, Tarbiat Modares University Tehran, Iran \\ ${ }^{2}$ Department of Genetics, Faculty of Medicine, Shahid Beheshti University of Medical Sciences, Tehran, Iran \\ "Corresponding author: Shirin Shahbazi, Department of Medical Genetics, Faculty of Medical Sciences, Tarbiat Modares University Tehran, 14115-111, \\ Tehran, Iran. Tel: +98 21 82884556, Fax: +98 21 82884555, E-mail: sh.shahbazi@modares.ac.ir
}

Received: 29 Jan. 2017; $\quad$ Revised: 6 Oct. 2017; Accepted: 11 Jan. 2018; Published online: 18 Apr. 2018

\begin{abstract}
Background: Coagulation Factor VII is a vitamin K-dependent serine protease which has a pivotal role in the initiation of the coagulation cascade. The congenital Factor VII deficiency is a recessive hemorrhagic disorder that occurs due to mutations of $F 7$ gene. In the present study C91S (p.C91S) substitution was detected in a patient with FVII deficiency. This mutation has not been characterized by a functional study.

Objectives: In this study, we aimed to evaluate the impact of C91S substitution on factor VII expression and function.

Materials and Methods: The $F 7$ complete cDNA was isolated from HepG2 cell line and inserted into the pcDNA3.1 mammalian expression vector. The desired mutation was generated by the site-directed mutagenesis and the wild-type and mutated constructs were transfected into $\mathrm{CHO}-\mathrm{K} 1$ cells. The protein activity and antigen level (antigen concentration) were validated in the culture medium and cell lysate of the transiently transformed cells. An immunocytochemistry procedure was also performed to evaluate the intracellular localization of the mutated and the wild-type FVII, as well.

Results: The present in vitro study has demonstrated that C91S antigen expression was increased in the transfected CHO-K1 cells compared to the wild-type (WT) protein. Despite an increased protein secretion, the factor VII coagulant activity was diminished following C91S substitution when it was assessed by a standard one-stage analysis. In addition, the immunocytochemistry procedure revealed that there was no difference in the intracellular localization of the C91S mutated FVII compared to the WT protein.

Conclusions: Our results present that C91S mutation has an effect on the coagulation activity, secretion, biosynthesis, and probably folding of the FVII leading to the FVII deficiency.

Keywords: Coagulation activity, F7 gene, Functional study, Site-directed mutagenesis
\end{abstract}

\section{Background}

The human FVII protein is a vitamin-k dependent glycoprotein which circulates in the plasma with a normal concentration of $500 \mathrm{ng} \cdot \mathrm{mL}^{-1}$. It is synthesized and secreted into the blood by the liver. The mature FVII molecule is a single chain protein composed of 406 amino acids and has a molecular weight of 50 $\mathrm{kD}$. This molecule is comprised of several discrete domains including the Gla domain (gamma-carboxy glutamic acid domain), two epidermal growth factor (EGF)-like domains, and a catalytic domain (serine protease) (1). FVII initiates extrinsic blood coagulation pathway. Upon vascular injury, FVII forms a complex with its receptor and cofactor; the tissue factor (TF) in the presence of calcium ion. Then, FVII in the complex is rapidly cleaved to its two-chained active form (FVIIa) and its catalytic activity multiplies so that FVIIa converts factor IX and X zymogens into active enzymes $(1,2)$. The complete $F 7$ gene (NM 000131) has been cloned and sequenced by O'Hara and colleagues in 1987 (3). It is localized on the 13q34 and comprises of the 9 exons spanning about $13 \mathrm{~kb}$ (3). The hereditary FVII deficiency (MIM 227500) is a rare autosomal recessive bleeding disorder with

Copyright (C) 2017 The Author(s); Published by National Institute of Genetic Engineering and Biotechnology. This is an open access article, distributed under the terms of the Creative Commons Attribution-NonCommercial 4.0 International License (http://creativecommons.org/licenses/ by-nc/4.0/) which permits others to copy and redistribute material just in noncommercial usages, provided the original work is properly cited. 
the variable clinical expressions that range from life-threatening to the very mild hemorrhages (4). This deficiency has an estimated incidence rate of 1 per 300,000-500,000 individuals in different populations $(5,6)$. There is a considerable molecular and phenotypic heterogeneity in the congenital FVII deficiency (7). A large number of molecular defects have been described in FVII-deficient patients that impair FVII biosynthesis and/or function $(7,8)$. The therapeutic strategies for the FVII deficiency are currently based on the frequent administration of the fresh frozen plasma or recombinant activated FVII. Therefore, elucidation of the molecular mechanisms of FVII deficiency by studying the effects of molecular defects in different functions of the gene products would help us to design new therapeutic strategies and protein engineering procedures (9).

The C91S (p.C91S) substitution was first reported in 2000 in a British patient with FVII deficiency (10). This substitution occurs in the exon 5 of $F 7$ gene and converts Cysteine 91 residue to a Serine in EGF2 (EGF-like 2) domain of the FVII protein. The EGF-like domains have been found to mediate protein-protein interactions. Experimental studies have shown that both the EGF-like and the serine protease domains are essential for the interaction between the tissue factor and FVII (11).

Unlike other coagulation factor deficiencies, the FVII deficiency has more complex phenotypic properties. In FVII deficiency there is not a direct association between coagulation tests findings and the clinical manifestations, therefore predicting clinical severity based on laboratory findings is almost impossible (7). Traditionally, functional studies have been conducted on plasma and with recombinant variants to provide molecular elements useful for defining and characterizing genetic alterations that lead to the FVII deficiency. Since there was a lack of previous functional studies to prove C91S pathogenicity and elucidating the effects of the mentioned substitution on the FVII properties, in the present study we cloned the wild-type and mutated FVII genes in pcDNA3.1/neo vector and expressed them in mammalian cells in order to be able to investigate the effects of C91S substitution on the functional properties of the FVII protein.

\section{Objectives}

The aim of the present study was to study the effect of the C91S substitution on the expression, secretion, intracellular localization, and coagulative activity of the FVII protein (FVIIC) and to obtain the functional evidence about C91S pathogenicity.

\section{Materials and Methods}

\subsection{Patient}

An informed consent was obtained from a patient with FVII deficiency and the blood sample was collected. The patient was a 20 years old male with mild bleeding symptoms (cutaneous symptoms, epistaxis, oral cavity bleeding) and FVII:C (IU.dL ${ }^{-1}$ ) 2\%. We detected C91S (c. $7807 \mathrm{G}>\mathrm{C}$ ) homozygous mutation upon sequencing of the patient's $F 7$ gene.

\subsection{Cell Culture and Construction of Expression Vectors}

As the source of normal human FVII mRNA, HepG2 cells were cultured in DMEM-high glucose (Gibco, United States) with 10\% FBS, 1\% penicillin/ streptomycin (Biosera, France) in a $5 \% \quad \mathrm{CO}_{2}$ atmosphere at $37{ }^{\circ} \mathrm{C}$ until reaching to the confluency. The total mRNA of the HepG2 cells was isolated using Trizol reagent (Life Technologies, United States) and cDNA was synthesized using RevertAid First Strand cDNA Synthesis kit (Thermo Fisher Scientific, United States). Isolation of FVII coding region was performed by PCR using a pair of specified primers with XhoI and EcoRI recognition sites: forward primer: AGAATTCTTCATCATGGTCTCCCAGG and reverse primer: TCTCGAGGCTAGGGAAATGGGGCTCG, respectively. The PCR product and pcCDNA3.1/neo mammalian expression vector were doubly digested with EcoRI and XhoI and purified using GENEJET PCR purification kit (Thermo Fisher Scientific, United States). The concentration of the purified $F 7$ gene and the linearized vector were measured and the fragment was inserted into the vector using Fast DNA ligation kit (Biobasic, Canada).

The resulting recombinant construct (pcDNA/WT), was transformed into DH5 $\alpha$ competent bacterial cells. The DH5 $\alpha$ competent cells were prepared using the $\mathrm{CaCl}_{2}$ method (12). After transformation, cells were cultured overnight on LB agar medium containing $100 \mu \mathrm{g} . \mathrm{mL}^{-1}$ ampicillin at $37{ }^{\circ} \mathrm{C}$. The transformed cells formed single colonies on agar medium. Several of these single colonies were amplified in LB broth medium (containing $100 \mu \mathrm{g} \cdot \mathrm{mL}^{-1}$ ampicillin) and plasmid DNA of each amplified colony was extracted using GeneJET plasmid miniprep kit (Thermo Fisher Scientific, United States) and sequenced using 3 single primers.

\subsection{Mutagenesis}

The C91S mutation was introduced into the pcDNA/WT vector using SOEing PCR site-directed mutagenesis. As described in Table 1, two pairs of overlapping primers 
Table 1. Primers used for the site-directed mutagenesis.

\begin{tabular}{cccc}
\hline Oligo name & Sequence $\left(\mathbf{5}^{\prime}\right.$ to $\left.\mathbf{3}^{\prime}\right)$ & Tm $\left({ }^{\circ} \mathrm{C}\right)$ & Amplicon (bp) \\
\hline Fmut & TGACCAGCTGATCTCTGTGAACGA & 63.62 & 1031 \\
Rbou & TGGCTGGCAACTAGAAGGCAC & 62.93 & \\
& & & \\
Fbou & AAGCTTGGTACCGAGCTCGGATC & 64.45 & 457 \\
Rmut & CCGCCGTTCTCGTTCACAGAGATC & 65.46 & \\
\hline
\end{tabular}

were designed and three independent PCR reactions were performed using pfu polymerase. The first reaction used Rbou and Fmut as primers and pcDNA/WT as the template to achieve a $1031 \mathrm{bp}$ product. The second reaction used Rmut and Fbou primers and pcDNA/ WT as the template to achieve a 457 bp product. In the third reaction, we used Fbou and Rbou primers and the product of the two previous reactions as the template. This reaction rejoined the $457 \mathrm{bp}$ and $1031 \mathrm{bp}$ products and resulted in a $1455 \mathrm{bp}$ fragment that was comprised of the mutated F7 gene and some parts of the pcDNA3.1 vector. The $1455 \mathrm{bp}$ fragment was doubly digested applying EcoRI and XhoI to achieve a 1348 bp fragment containing $F 7$ gene. The 1348 bp fragment was then ligated into the double digested pcDNA3.1/ neo vector using fast DNA ligation kit. Further sequencing was conducted to verify the presence of the C91S alteration in the resulting recombinant construct (pcCDNA/C91S).

\subsection{CHO-K1 Cell Culture and Transfection}

Mammalian cells of choice to express $F 7$ gene were CHO-K1 cells. These cells do not secrete blood coagulation proteins but are able to efficiently carry out the normal processing events and post-translational modifications occurring in the liver (13-15). CHO-K1 cells were cultured in DMEM-F12 medium (Gibco, United States) supplemented with the 10\% FBS plus $1 \%$ penicillin/streptomycin (Biosera, France) in a $5 \%$ $\mathrm{CO}_{2}$ atmosphere at $37{ }^{\circ} \mathrm{C}$ in a T25 flask to proliferate. 400000 cells were counted and subcultured in 60 $\mathrm{mm}$ plates $24 \mathrm{~h}$ prior to transfection. The cells were transfected with the pcDNA/C91S and pcDNA/WT vectors in the $60 \mathrm{~mm}$ plates using Turbofect transfection reagent (Thermo Fisher Scientific, United States) based on the manufacturer's protocol. To estimate the fidelity of transfection, a third vector carrying GFP reporter was also applied. $48 \mathrm{~h}$ after transfection, the conditioned medium of each dish were collected. Then, the cells of each transfection were trypsinized and lysed using a freeze-thaw protocol. The collected conditioned media and lysates were kept at $-70{ }^{\circ} \mathrm{C}$ until analysis.

\section{5. mRNA Study}

To confirm the successful transfection and FVII expression in CHO-K1 cells, total RNA of transfected cells was also extracted using Trizol reagent (Life Technologies, United States). The cDNA was synthesized (RevertAid First Strand cDNA Synthesis kit, Thermo Fisher Scientific, United States) and an RT-PCR was performed to amplify human $F 7$ mRNA. The following primers were used in RT-PCR reaction: FVIICD2-F: TGTGTGAACGAGAACGGCG and FVIICD2-R: ACCTTCCGTGACTGCTGC.

\subsection{Protein Expression and Coagulant Activity Measurement}

FVIIC was determined by a one-stage PT-based method on the collected conditioned media and cell lysates. This procedure was performed using an automated Sysmex CA-1500 Coagulation Analyzer System. The FVII secretion and its intracellular level were evaluated by the measurement of FVII antigen in the collected conditioned media and cell lysates using ELISA method (Factor VII human ELISA kit Abcam Cat\#ab108829, United Kingdom). The standard curve was constructed applying a serial dilution of the standard recombinant human FVII. This assay was performed in duplicate for all standard samples and test samples. The conditioned medium and cell lysate of untransfected CHO-K1 cells were used as negative control for this test.

\subsection{Immunocytochemistry}

In order to study intracellular localization of the wild-type and mutated FVII protein, we conducted an immunocytochemistry test on CHO-K1 cells transfected with the wild-type and the mutated FVII, respectively. To this purpose, CHO-K1 cells were grown overnight on the glass coverslips and transfected as described earlier. The coverslips were washed once in PBS and fixed in the 3\% paraformaldehyde in PBS for $1 \mathrm{~h}$. The cells were washed 3 more times and permeabilized in $0.1 \%$ triton X100 (Sigma, United States) for $5 \mathrm{~min}$. After further washing, we blocked the coverslips in $1 \%$ BSA in PBS for $30 \mathrm{~min}$. Then, 
rabbit anti human factor 7 antibody $\left(5 \mu \mathrm{g} \cdot \mathrm{mL}^{-1}\right.$ in PBS containing 1\% BSA, Abcam Cat\# ab97614, United Kingdom) was added to each coverslip and incubated for $1 \mathrm{~h}$ at room temperature. The cells were again washed 3 times with the PBS and then incubated with DyLight ${ }^{\circledR} 488$ goat anti-rabbit $\operatorname{IgG}(\mathrm{H}+\mathrm{L}$, at a dilution of 1/300 in PBS containing 1\% BSA; Abcam cat\# ab96899, United Kingdom) for 30 minutes. After further washing, the coverslips were mounted onto glass slides and were studied under a fluorescent microscope (Olympus).

\section{Results}

\subsection{Constructs and Mutagenesis}

pcDNA/WT and pcDNA/C91S sequencing analysis showed the desired direction of the $F 7$ gene with no unwanted changes in the sequence of the gene and vector. The C91S (c.7807G $>$ C) mutation was successfully created on pcDNA/C91S (Fig. 1). The parallel CHO-K1 transfection by pcDNA/GFP showed an appropriate rate of transfection.

\section{2. $m R N A$ Expression}

RT-PCR showed a 680 bp fragment, suggesting that the transfected cells expressed wild-type and mutated


Figure 1. The sequencing results showing the successful introduction of the C91S mutation against the wild-type construct. Arrow indicates the substituted nucleotides. human $F 7$ genes (Fig. 2) and the transfection had been performed successfully.

\subsection{Antigen Assay}

Upon sketching the standard curve, FVII antigen assay of the conditioned medium and the cell lysates revealed an elevated expression of the mutated FVII compared to the WT transfected cells. The conditioned medium of the C91S transfected cells showed 3 folds increase in the level of FVII antigen. The level of antigen in the cell lysate of the C91S substitution transfected cells was 1.5 fold more than that of WT (Table 2).

\subsection{Coagulation Activity}

Another functional property of FVII that may be affected by the mutation is its coagulative activity. We studied this function by running a PT-based test on supernatants and lysates collected from transfected cells. The results of this test revealed that in spite of an increased antigen level, FVII with the C91S mutation had undetectable coagulative activity in both cell supernatant and lysate compared to the coagulative activity of the WT transfected cells (as considered 100\%) (Table 2).

\subsection{Immunocytochemistry}

Using immunofluorescent staining to detect intracellular FVII antigens, we found no difference between intracellular localization of the wild-type and mutated FVII (Fig. 3). The staining pattern of the WT and C91S FVII was mostly perinuclear, with some diffuse signal throughout the cell cytoplasm, suggesting that the C91S substitution does not cause the FVII protein to retain in any cellular compartments.

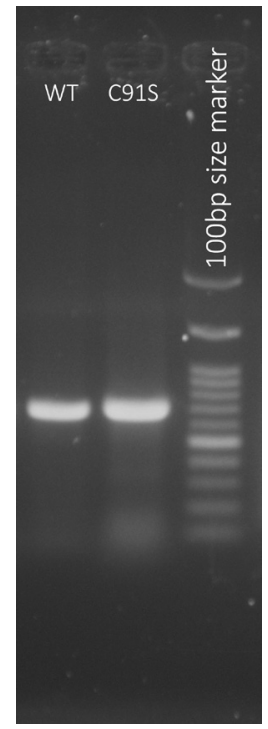

Figure 2. RT-PCR performed on the cDNA obtained from the transfected cells. Agarose gel electrophoresis shows a 680 bp band. 
Table 2. Transient expression assays of the FVII WT and FVII C91S in CHO-K1 cells. The table illustrates the results of FVII antigen and coagulant activity measurements in the cell lysates and cell supernatants of the transiently transfected cells. The FVII level measurement in the cell lysates and in the conditioned media 48 hours after transfection of the cells with each construct.

\begin{tabular}{ccc}
\cline { 2 - 3 } & FVII WT & FVII C91S \\
\hline Supernatant (VII:Ag) & $23.84 \mathrm{ng} . \mathrm{mL}^{-1}$ & $73.3 \mathrm{ng} \cdot \mathrm{mL}^{-1}$ \\
Cell lysate (VII:Ag) & $142.65 \mathrm{ng} \cdot \mathrm{mL}^{-1}$ & $218.28 \mathrm{ng} \cdot \mathrm{mL}^{-1}$ \\
Supernatant (VII:C) & $100 \%$ & $\mathrm{ND}$ \\
Cell lysate (VII:C) & $100 \%$ & $\mathrm{ND}$ \\
\hline
\end{tabular}

ND: not detectable.

\section{Discussion}

The most severe cases of the FVII deficiency are either homozygous or compound heterozygous for the deleterious mutations resulting in the FVII:C level to an amount of less than $2 \%$ of the normal condition (7). Here, we have reported a patient homozygous for the Cys91Ser substitution which is caused by the c. $7807 \mathrm{G}>\mathrm{C}$ mutation in the exon 5 . The $\mathrm{C} 91 \mathrm{~S}$ residue is a highly conserved amino acid that has been unchanged in the course of evolution between different species (Fig. 4). There are several conserved cysteine residues in the structure of FVII as well as other vitamin-K dependent factors which play essential roles in the activity, secretion, and 3D structure of these proteins by forming disulfide bonds. Mutations in either of these amino acids will have a major effect on the protein structure. The EGF2 domain contains 6 cysteines

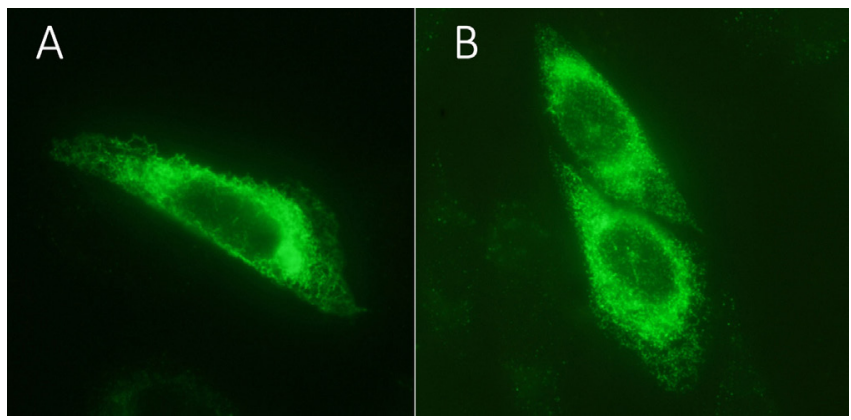

Figure 3. Immunostaining of the WT (A) and C91S (B) transfected $\mathrm{CHO}-\mathrm{K} 1$ cells showing the perinuclear pattern of FVII staining.

that are arranged to form 3 disulfide bonds. One of these disulfide bonds is located between cysteine 91 and cysteine 102 that could be disrupted due to C91S substitution and severely impair the structural integrity of the FVII protein $(16,17)$. Therefore, this mutation could be considered as a basis for the severe dysfunction of the enzyme. However, the patient did not exhibit severe complications such as hemarthrosis or gastrointestinal bleeding. So, the main purpose of the present study was to demonstrate whether or not the C91S is a disease-causing mutation.

By FVII antigen measurement in the conditioned medium, we showed that the C91S mutation affects the secretion and causes the mutated FVII to be secreted in higher levels compared to the wild-type FVII protein. Evaluation of the FVII antigen in the cell lysate also revealed that the mutated FVII has a higher intracellular level than that of wild-type FVII. The increased intracellular level of FVII has been previously reported

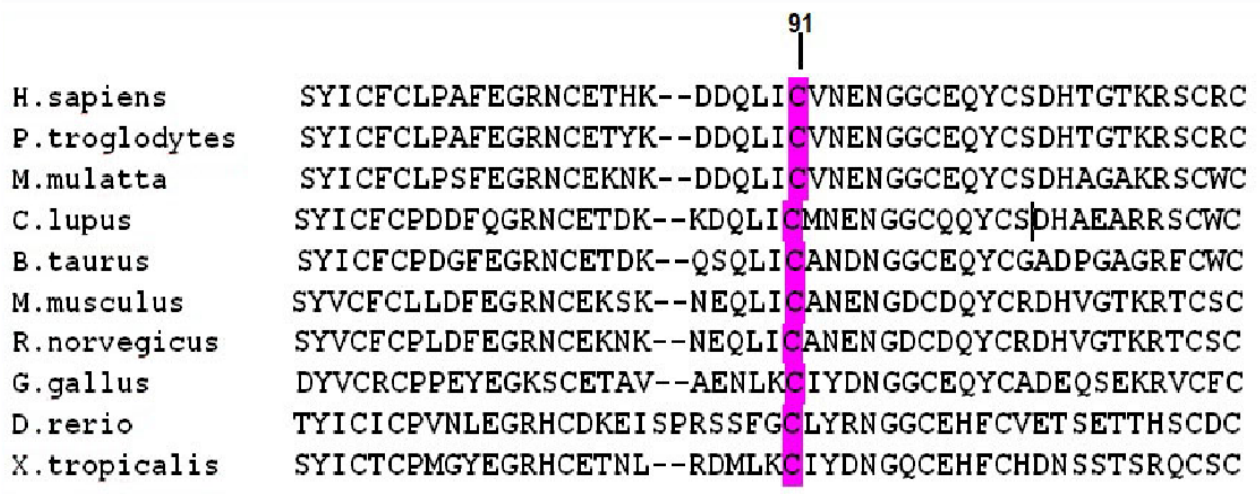

Figure 4. The conservation of the C91 residue in the second EGF-like domain. The multiple alignment analysis was used to align FVII proteins from different species for this domain containing the C91S mutation. 
for the several mutated variants. It has been shown that the intracellular level of Thr359Met and Cys22Arg increases due to an impaired secretion $(18,19)$. But, unlike C91S in both cases, the extracellular level of FVII was simultaneously reduced. Therefore, it can be deduced that an increased intracellular level of the C91S variant is not due to an impaired secretion, but, it might be due to the increased biosynthesis or a folding problem. For a number of proteins, it has been shown that mutations which lead to the loss of disulfide bonds can cause the protein to fold with a decreased rate, resulting in accumulation of the protein in the cells (20). On the other hand, binding of secretory proteins (such as coagulation factors) to BiP (Binding Immunoglobulin Protein) chaperon can affect secretion rate, intracellular accumulation, and biosynthesis of these proteins (18, 21, 22). In addition, as C91S substitution occurs in the coding region and the expression of the cloned $F 7$ gene is under control of a strong CMV promoter; it might be suggested that the increased level of mutated FVII protein occurs in the translational or post-translational steps of the expression instead of transcription.

In spite of an increased FVII level in the conditioned medium of the cells transfected with the defective FVII, coagulant activity was undetectable suggesting that C91S substitution does severely affect FVII structure and activity. These results are consistent with the previous studies showing that EGF2 mutations impair FVII coagulant activity. EGF-like domains have an important role in the FVII activity, structure and proteinprotein interactions and amino acid substitutions of these domains have destructive effects on the enzyme activity. In a study by D'Andrea et al., it has been revealed that occurrence of Arg110Cys and Asp123Tyr mutations; both of which in the EGF2 domain, cause a severe coagulant activity reduction (23). Similarly, in another study by Hunault et al., functional study of Gly97Cys and Gln100Arg mutations also showed a reduced coagulant activity of the mutated proteins (1). It can be hypothesized that the decreased activity observed in the presence of C91S mutation could be due to severe conformational changes and reduced interaction between FVII and tissue factor $(11,24)$.

We used immunocytochemistry method to localize intracellular WT and mutated FVII. This method showed that there was no difference in the cellular localization between WT and the mutated FVII. Staining of the FVII was mostly perinuclear with some diffuse staining all over the cell cytoplasm, showing that the wild-type and the mutated FVII are biosynthesized normally and accumulate in the endoplasmic reticulum and Golgi apparatus just prior to the secretion in order to achieve folding and post-translational modifications $(1,17)$. This pattern has been previously observed for the Gly97Arg mutation in EGF2 (1) and Ser190Phe mutation in the catalytic domain (25). A normal pattern of staining and lack of difference between WT and C91S suggests that the mutation doesn't impair transportation of mutated FVII through the secretory pathway.

\section{Conclusions}

From this, we conclude that C91S mutation affects FVII biosynthesis and/or folding that causes a higher intracellular level of the protein. As a result of FVII intracellular accumulation, more defective FVII molecules are secreted into extracellular space and cause an increased level of FVII antigen. This conclusion is consistent with the phenotypic characteristics of the patient in whom C91S substitution was found, meaning that increased level of secreted mutated FVII causes the patient to have the minimal FVII:C, sufficient for the initiation of coagulation cascade leading to the mild bleeding symptoms despite a severe loss of coagulation activity (FVII:C 2\%) (26). The unrecognized variants within the FVII gene locus and/or related gene loci which may modulate the FVII gene expression or protein prossesing in vivo should be considered in the phenotypic manifestations of the patient.

We have found a naturally occurring missense mutation within the second EGF-like domain that affects the coagulation activity, secretion, biosynthesis and probably folding of the FVII that leads to the FVII deficiency. Therefore the present study provides a functional evidence showing that C91S substitution is a disease-causing mutation. This further suggests the importance of the second EGF-like domain in the FVII functions.

\section{Acknowledgments}

This work was supported by the grants from Iran National Science Foundation (INSF) to Dr. Shirin Shahbazi and Research deputy of Tarbiat Modares University to Amir Mashayekhi. The authors would like to state that they have no specific interest which might pose a conflict or bias.

\section{References}

1. Hunault M, Arbini AA, Carew JA, Peyvandi F, Bauer KA. Characterization of two naturally occurring mutations in the second epidermal growth factor-like domain of factor VII. Blood. 1999;93(4):1237-1244.

2. Tanaka R, Nakashima D, Suzuki A, Miyawaki Y, Fujimori $\mathrm{Y}$, Yamada $\mathrm{T}$, et al. Impaired secretion of carboxyl-terminal truncated factor VII due to an F7 nonsense mutation associated with FVII deficiency. Thromb Res. 2010;125(3):262-266. doi: 
10.1016/j.thromres.2009.09.014.

3. O'Hara PJ, Grant FJ, Haldeman BA, Gray CL, Insley MY, Hagen FS, et al. Nucleotide sequence of the gene coding for human factor VII, a vitamin K-dependent protein participating in blood coagulation. PNAS. 1987;84(15):5158-5162.

4. Cooper DN, Millar DS, Wacey A, Banner DW, Tuddenham EG. Inherited factor VII deficiency: molecular genetics and pathophysiology. Thromb Haemost. 1997;78(1):151-160.

5. Peyvandi F, Duga S, Akhavan S, Mannucci PM. Rare coagulation deficiencies. Haemophilia. 2002;8(3):308-321.

6. Palla R, Peyvandi F, Shapiro AD. Rare bleeding disorders: diagnosis and treatment. Blood. 2015;125(13):2052-2061. doi: 10.1182/blood-2014-08-532820.

7. McVey JH, Boswell E, Mumford AD, Kemball-Cook G, Tuddenham EG. Factor VII deficiency and the FVII mutation database. Hum Mutat. 2001;17(1):3-17. doi: 10.1002/1098-1004(2001(17:1<3::AID-HUMU2>3.0.CO;2-V.

8. Mariani G, Herrmann FH, Bernardi F, Schved JF, Auerswald $\mathrm{G}$, Ingerslev J. Clinical manifestations, management, and molecular genetics in congenital factor VII deficiency: the International Registry on Congenital Factor VII Deficiency (IRF7). Blood. 2000;96(1):374.

9. Furie B, Furie BC. Molecular and cellular biology of blood coagulation. $N$ Engl J Med. 1992;326(12):800-806. doi: 10.1056/NEJM199203193261205.

10. Millar DS, Kemball-Cook G, McVey JH, Tuddenham EG, Mumford AD, Attock GB, et al. Molecular analysis of the genotype-phenotype relationship in factor VII deficiency. Hum Genet. 2000;107(4):327-342.

11. Chang JY, Stafford DW, Straight DL. The roles of factor VII's structural domains in tissue factor binding. Biochemistry. 1995;34(38):12227-12232.

12. Dagert M, Ehrlich SD. Prolonged incubation in calcium chloride improves the competence of Escherichia coli cells. Gene. 1979;6(1):23-8.

13. Kaufman RJ. Selection and coamplification of heterologous genes in mammalian cells. Methods Enzymol. 1990;185:537-66.

14. Xiao W, Li CQ, Xiao XP, Lin FZ. Expression and fast preparation of biologically active recombinant human coagulation factor VII in CHO-K1 cells. Genet Mol Res. 2013;12(4):6813-6824. doi: 10.4238/2013.December.16.7.

15. Sutkeviciute I, Mistiniene E, Sereikaite J, Bumelis VA. The influence of different glycosylation patterns on factor VII biological activity. Biochimie. 2009;91(9):1123-1130. doi: 10.1016/j.biochi.2009.05.015.

16. Branchini A, Rizzotto L, Mariani G, Napolitano M, Lapecorella
M, Giansily-Blaizot $\mathrm{M}$, et al. Natural and engineered carboxy-terminal variants: decreased secretion and gainof-function result in asymptomatic coagulation factor VII deficiency. Haematologica. 2012;97(5):705-709. doi: 10.3324/ haematol.2011.049403.

17. Kaufman RJ. Post-translational modifications required for coagulation factor secretion and function. Thromb Haemost. 1998;79(6):1068-1079.

18. Arbini AA, Mannucci M, Bauer KA. A Thr359Met mutation in factor VII of a patient with a hereditary deficiency causes defective secretion of the molecule. Blood. 1996;87(12):50855094.

19. Fromovich-Amit Y, Zivelin A, Rosenberg N, Tamary H, Landau M, Seligsohn U. Characterization of mutations causing factor VII deficiency in 61 unrelated Israeli patients. $J$ Thromb Haemost. 2004;2(10):1774-1781. doi: 10.1111/j.15387836.2004.00921.x.

20. Yokota A, Izutani K, Takai M, Kubo Y, Noda Y, Koumoto Y, et al. The transition state in the folding-unfolding reaction of four species of three-disulfide variant of hen lysozyme: the role of each disulfide bridge. J Mol Biol. 2000;295(5):1275-1288. doi: 10.1006/jmbi.1999.3442.

21. Orlova NA, Kovnir SV, Vorobiev, II, Gabibov AG, Vorobiev AI. Blood Clotting Factor VIII: From Evolution to Therapy. Acta Naturae. 2013;5(2):19-39.

22. Fantacini DM, Fontes AM, de Abreu Neto MS, Covas DT, Picanco-Castro V. The F309S mutation increases factor VIII secretion in human cell line. Rev Bras Hematol Hemoter. 2016;38(2):135-140. doi: 10.1016/j.bjhh.2016.04.002.

23. D'Andrea G, Bossone A, Lupone MR, Peyvandi F, Maisto G, Perricone F, et al. Molecular characterization of a factor VII deficient patient supports the importance of the second epidermal growth factor-like domain. Haematologica. 2004;89(8):979984.

24. Edgington TS, Dickinson CD, Ruf W. The structural basis of function of the TF. VIIa complex in the cellular initiation of coagulation. Thromb Haemost. 1997;78(1):401-405.

25. Jiang M, Wang Z, Yu Z, Bai X, Su J, Cao L, et al. Anovel missense mutation close to the charge-stabilizing system in a patient with congenital factor VII deficiency. Blood Coagul Fibrinolysis. 2011;22(4):264-270. doi: 10.1097/MBC.0b013e3283447388.

26. Giansily-Blaizot M, Aguilar-Martinez P, Biron-Andreani C, Jeanjean P, Igual H, Schved JF. Analysis of the genotypes and phenotypes of 37 unrelated patients with inherited factor VII deficiency. Eur Hum Genet. 2001;9(2):105-112. doi: 10.1038/ sj.ejhg.5200593. 